\title{
Magnetic moment in Ni clusters estimated by an electronic-shell model
}

\author{
N. Fujima and T. Yamaguchi \\ Faculty of Engineering, Shizuoka University, Hamamatsu 432, Japan
}

(Received 13 December 1995)

\begin{abstract}
We estimate the size dependence of the magnetic moment of $\mathrm{Ni}$ clusters by using a simple electronic shell model, in which the $4 s$ electron levels are described by the spherical harmonic oscillator model. This model reproduces the whole structure in the size dependence of the magnetic moment which has been experimentally observed. [S0163-1829(96)06926-3]
\end{abstract}

The magnetism of small ferromagnetic transition-metal clusters has been widely studied because of their extraordinary magnetic moments. Recent experiments have revealed that the magnetic moments of ferromagnetic transition-metal clusters align in superparamagnetic order under a certain thermal equilibrium condition and that the average magnetic moments per atom are larger than the bulk moments and gradually, but nonmonotonously approach the bulk moments with increasing cluster size. ${ }^{1-3}$

Apsel et al. measured the magnetic moments of Ni clusters by using the Stern-Gerlach method, and clarified the size dependence of the magnetic moments of $\mathrm{Ni}_{N}$ clusters $(N<200)$ with the size resolution as the number of atoms of 1. ${ }^{4}$ Besides the gradual decrease, they found some structures in the size dependence, that is, peaks at $N=8,20,42$, and 71 and $\operatorname{dips}$ at $N=6,13,34$, and 56 (see Fig. 3).

They explained these structures by the cluster geometry, where the surface atoms or atoms with a small coordination number have larger magnetic moments than those of the inner atoms with a larger coordination number. Of course, the geometrical environments are very important to determine the local magnetic moment in a cluster, and actually some structures in size dependence can be explained by the geometries. However, it seems that the whole structure in the size dependence has not been explained completely only by the geometries.

In the present paper, we estimate the size dependence on the magnetic moment of $\mathrm{Ni}$ clusters by using a simple electronic-shell model, and explain the whole structure of the size dependence which has been experimentally observed. The fundamental idea has already been published. ${ }^{5}$

Electronic states of $\mathrm{Ni}$ clusters calculated by the local spin-density-functional method show a typical energy level scheme, that is, the $3 d$-electron-derived levels, which are located densely around the highest occupied level, and the $4 s$-electron-derived levels, which are located sparsely both under and above the $3 d$-electron-derived levels. ${ }^{5}$ The $4 s$-electron-derived levels do not distribute uniformly, but are bunching and constitute some group of levels when the cluster is almost spherical or highly symmetrical.

Figure 1 shows a schematic diagram of energy levels in Ni clusters. An increase of cluster size reduces the energy interval between the bunches of the $4 s$ electron levels, and increases the number of bunches appearing under the $3 d$ electron levels.
If some $4 s$ electron levels exist below the $3 d$ electron levels, holes exist at the top of $3 d$ electron levels. The number of holes is equal to the number of $4 s$ levels below the $3 d$ electron levels, since the number of valence electrons in a $\mathrm{Ni}$ atom, 10, is equal to the degeneracy of the $3 d$ electron levels. The number of holes is equal to the number of unpaired electrons, that is, the amount of spin magnetic moments (in Bohr magnetons $\mu_{B}$ ). Thus, if the $4 s$ levels are bunched well, it is expected that the magnetic moment increases stepwise at a cluster size where the $4 s$ levels are filled. The electronic-state calculation by the local spindensity-functional method has predicted that such a stepwise change occurs at $N=8$ and 19 in $\mathrm{Ni}_{N}$ clusters. ${ }^{5}$ Based on the results of the calculation, we estimate the size dependence of the magnetic moment in the region of larger size by using the following simple model.

We assume that the electronic energy levels of Ni clusters consist of both bandlike $3 d$-electron levels which are located around the highest occupied level and the $4 s$ electron levels which distribute discretely. Figure 2 shows the schematic

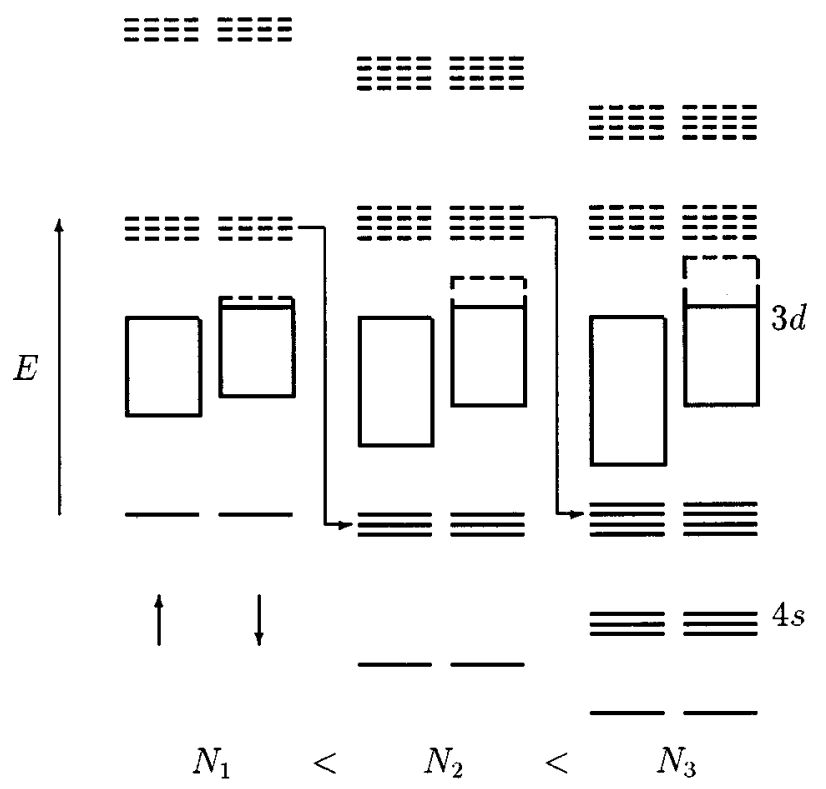

FIG. 1. Schematic diagram of the energy levels in $\mathrm{Ni}_{N}$ clusters $\left(N_{1}<N_{2}<N_{3}\right)$. Solid and dashed lines indicate occupied and unoccupied levels, respectively. 


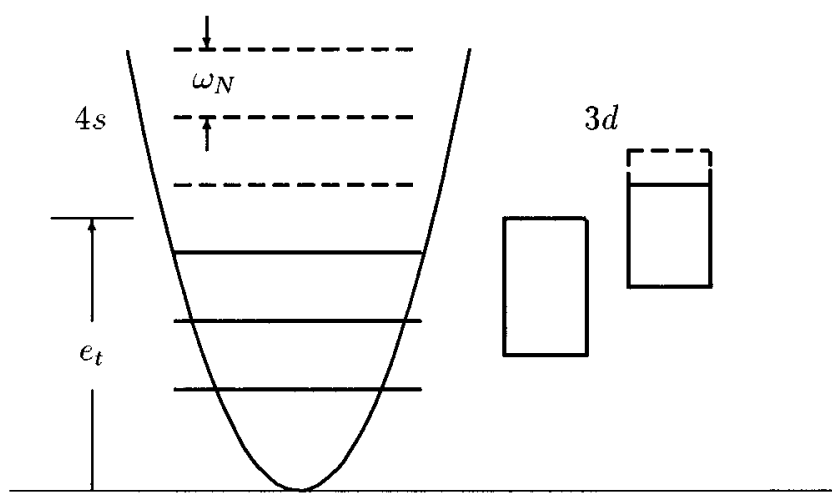

FIG. 2. Electronic-shell model for estimating magnetic moment in $\mathrm{Ni}$ clusters.

diagram of our model. We take the spherical harmonic oscillator model for the energy levels of the $4 s$ electrons, $E_{n}$ [in atomic units, (a.u.)],

$$
E_{n}=(n+3 / 2) \omega_{N} \quad(n=0,1,2, \ldots) .
$$

Here, $\omega_{N}$ is the oscillation frequency, which determines the dimension of the clusters and varys with the number of atoms, $N$. The $4 s$ electron level is occupied if the energy $E_{n}$ is lower than a threshold energy $e_{t}$. The number of $4 s$ electrons in a cluster, $n_{4 s}$, is given by the equation

$$
n_{4 s}=\frac{1}{3}\left(n_{\mathrm{occ}}+1\right)\left(n_{\mathrm{occ}}+2\right)\left(n_{\mathrm{occ}}+3\right)
$$

where $n_{\mathrm{occ}}$ is the quantum number of the highest occupied level.

According to the assumption, holes of the same number as the number of $4 s$ electrons appear at the top of the $3 d$ electron levels. Then, we estimate the magnetic moment of the clusters from the number of holes, i.e., number of unpaired electrons. The magnetic moment per atom, $\mu_{N}$, is given by

$$
\mu_{N}=n_{4 s} / N
$$

The oscillation frequency $\omega_{N}$ is expected to depend on the volume of clusters as

$$
\omega_{N}=e_{b}(3 N)^{-1 / 3}
$$

To estimate the magnetic moment, we determine two parameters $e_{b}$ and $e_{t}$. We choose a value of the bandwidth of the bulk crystal (the width from the bottom of the band to the Fermi level: 0.322 a.u.) for $e_{b}$ and a value of the width from the bottom to a value between the Fermi level and the center of the $3 d$ band (0.290 a.u.) for $e_{t}$. The fact that $e_{t} \sim e_{b}$ means that the quantum number of the highest occupied level is expressed as

$$
n_{\mathrm{occ}}+\frac{3}{2} \sim(3 N)^{1 / 3}
$$

It is noted that, by solving Eq. (2) for a given value $n_{4 s}=n_{e}$, the quantum number for which a shell closing occurs is expressed by

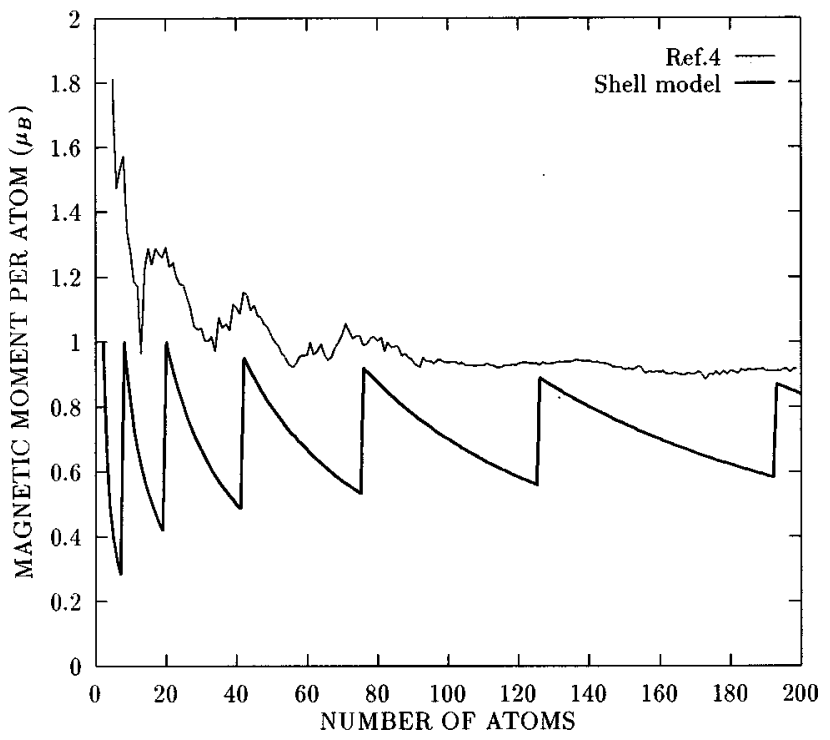

FIG. 3. Magnetic moments per atom in Ni clusters $\left(\mu_{B}\right)$. Thick and thin lines indicate the estimation in the present work and the experimental results (Ref. 4), respectively.

$$
n_{\mathrm{occ}}+\frac{3}{2} \approx\left(3 n_{e}\right)^{1 / 3}+\frac{1}{3}\left(3 n_{e}\right)^{-1 / 3}-\frac{1}{2} .
$$

Then, Eqs. (5) and (6) give the fact that the number of $4 \mathrm{~s}$ electrons per atom at the shell closing is estimated to be nearly equal to 1 , i.e., $N \sim n_{e}$.

Figure 3 shows the estimation of the size dependence of the magnetic moment in $\mathrm{Ni}$ clusters by using the above model and parameters. For comparison, recent experimental results ${ }^{4}$ are also shown. It is found that the estimation reproduces the prominent four peaks experimentally observed around at $N=8,20,40$, and 70. Moreover, two weak broad peaks observed around at $N=135$ and 190 also appear in the estimation at $N=125$ and 190 . Therefore, it is found that the estimation can reproduce the observed peaks, which appear at the number roughly corresponding to the shell-closing numbers. Because the degeneraces of the energy levels are overestimated in the simple shell model, the higher two peaks in the estimation are excessively prominent; if an inharmonic term $\left(\propto r^{4}\right)$ and/or an anisotropic term which remove the high degeneracy of the levels are taken into the model, the fine structures and the broadness of the observed peaks would be reproduced better. The fact that the higher two peaks $(N=135$ and 190) appear in a larger number of atoms than the corresponding shell-closing numbers $\left(n_{e}=\right.$ 112 and 168) suggests that the number of the $4 s$ electrons per atom decreases gradually with cluster size and becomes close to the bulk value $(\sim 0.6)$.

Positions and heights of the peaks are determined mainly by the relative value of $e_{t}$ to $e_{b}$, and are not sensitive to the absolute values of $e_{t}$ and $e_{b}$. With increasing (decreasing) $e_{t}$ relatively to $e_{b}$, the number of $4 s$ electrons per atom increases (decreases). As a result, the peak positions become smaller (larger) and the heights larger (smaller). For example, peak positions and heights change approximately by $5 \%$ when the value of $e_{t}$ changes by \pm 0.005 from 0.290 
(a.u.) with a fixed value of $e_{b}$. However, the general feature of the structure does not change.

The absolute values of the magnetic moments by the estimation $\left(\leqslant 1 \mu_{B}\right)$ are smaller than experimental values, especially in the region of small size. It is due to the estimation that the number of $4 s$ electrons is nearly equal to 1 at the shell-closing number. However, it is noted that theoretical studies using the local spin-density-functional method ${ }^{5-9}$ show that the magnetic moments are similar to our estimation rather than the experimental results. Moreover, the calculation by using the nonlocal spin-density-functional method (the generalized gradient approximation ${ }^{10,11}$ ) shows similar results. ${ }^{12}$ These calculations were performed with fixed atomic structures. A structure-optimized calculation might give larger magnetic moments in the region of small size.
In conclusion, we estimate the size dependence of the magnetic moment in $\mathrm{Ni}$ clusters by using the simple electronic-shell model. The estimation reproduces the prominent peaks in the size dependence of the magnetic moment which are experimentally observed. We believe that the estimation can be applied to other ferromagnetic transitionmetal clusters $\mathrm{Fe}_{N}$ and $\mathrm{Co}_{N}$, where the magnetic moments per atom $\mu_{N}$ are given by the formula

$$
\mu_{N}=\left(10-n_{b}\right)+\left(n_{\mathrm{occ}}+1\right)\left(n_{\mathrm{occ}}+2\right)\left(n_{\mathrm{occ}}+3\right) / 3 N .
$$

Here, $n_{b}$ is the number of valence electrons per atom, i.e., 8 for Fe clusters and 9 for Co clusters.
${ }^{1}$ D. C. Douglass, A. J. Cox, J. P. Bucher, and L. A. Bloomfeld, Phys. Rev. B 47, 12874 (1993).

${ }^{2}$ I. L. M. Billas, A. Châtelain, and W. A. de Heer, Science 265, 1682 (1994).

${ }^{3}$ S. N. Khanna and S. Linderoth, Phys. Rev. Lett. 67, 742 (1991).

${ }^{4}$ S. E. Apsel, J. W. Emmert, J. Deng, and L. A. Bloomfeld, Phys. Rev. Lett. 76, 1441 (1996).

${ }^{5}$ N. Fujima and T. Yamaguchi, J. Phys. Soc. Jpn. 58, 3290 (1989).

${ }^{6}$ R. P. Messmer, S. K. Knudson, K. H. Johnson, J. B. Diamond, and C. Y. Yang, Phys. Rev. B 13, 1396 (1976).
${ }^{7}$ H. Basch, M. D. Newton, and J. W. Moskowitz, J. Chem. Phys. 73, 4492 (1980).

${ }^{8}$ K. Lee, J. Callaway, K. Kwong, R. Tang, and A. Ziegler, Phys. Rev. B 31, 1796 (1985).

${ }^{9}$ G. F. Holland, D. E. Ellis, and W. C. Trogler, J. Chem. Phys. 83, 3507 (1985).

${ }^{10}$ J. P. Perdew and Y. Wang, Phys. Rev. B 33, 8800 (1986).

${ }^{11}$ J. P. Perdew and Y. Wang, Phys. Rev. B 45, 13244 (1992).

${ }^{12}$ N. Fujima, N. Sasaki, and T. Yamaguchi (unpublished). 